\title{
Dimensioning fiber delay lines in optical burst switching networks
}

\author{
G.M. Li, Z.K. Sun, S.H. Geng \\ School of Information Engineering \\ SHANDONG University at WEIHAI, China
}

\begin{abstract}
We investigate two different node structures with fiber delay lines (FDLs) in optical burst switching networks: FDL share per node and FDL share per link. We study the roles of maximum delay and the single FDL element granularity in FDL share per node. Our simulations show that FDLs with limited delay can effectively improve the network performance in terms of burst loss probability, but increasing the maximum delay beyond a certain range or making FDL granularity much finer cannot improve network performance effectively. The performance improvements in different reservation schemes are also compared.
\end{abstract}

Keywords- optical burst switching; Optical buffer

\section{INTRODUCTION}

Optical communication systems will play an important role in the next generation network. At present, there are three major candidate solutions for optical communication systems based on wavelength-division multiplexing (WDM) technology: optical circuit switching (OCS), optical packet switching (OPS) and optical burst switching (OBS). Given the limitations of current optical processing capability and lack of practical optical buffers, OBS is considered to be the top choice for the next generation network in the near term since it is more efficient than OCS in terms of wavelength utilization efficiency and it does not require the technological breakthroughs needed to make OPS a reality. In OBS networks, the payload is configured in the form of data bursts and kept in the optical domain. Each optical burst has an associated control packet (CP). CP is sent in a separate control channel and processed electronically in order to schedule a pending data burst to an output port [1]. The scheduling algorithm may be First-Fit (FF), Latest available unscheduled channel (LAUC), First-Fit with void filling (FFVF) or Latest available unscheduled channel with void filling (LAUC-VF) [2]. They differ in bandwidth utilization and computational complexity. Another key characteristic is that OBS uses one-way reservation, i.e., an optical data burst (DB) starts transmission with an offset period after the control packet is sent, without waiting for positive acknowledgement of the end-to-end path setup. Due to the one way reservation mechanism, burst contention resolution is very important to improve network performance.

In general, burst loss can be categorized into two types in OBS networks: 1) control packet loss; a control packet may be lost on the route or control packet congestion may occur in the outgoing control channel or inside the control packet processor; 2) congestion occurs in the output port of data channels. Most existing research focus on the second type of loss and assume there is no control packet loss or congestion. To resolve this type of burst loss, three major options can be applied: 1) wavelength domain, in which a node with wavelength conversion capability is considered [3]; 2) space domain, in which a contending data burst can be sent along a different route to the destination using deflection routing [4];3) time domain, in which burst segmentation drops the contending portion of the data burst to improve the bandwidth utilization [5-7]. Another time domain solution is to use FDLs to delay the arrival of data bursts in order to resolve contention. In this paper, we focus on using FDLs to resolve burst contention in OBS networks. Although optical buffers are not mandatory in OBS networks, some studies have shown limited FDLs in OBS networks can effectively improve the network performance [12, 8-11]. In [1,8], FDLs are used to improve the extendedoffset-time based Quality of service (QoS) scheme. Rather than considering FDL one by one, [2] partitions FDLs into groups and simplifies the scheduling algorithms. [9] develop analytical models to evaluate the performance of a single node with FDLs. In [10], burst rescheduling is proposed in which a simple nonvoid filling scheduling algorithm is used to achieve performance comparable to the more complex void-filling algorithm. At the same time, FDLs are used to reschedule bursts to resolve burst contentions. However, FDL assignment is limited to bursts which traverse the last hop. In all existing research mentioned above, each node is assumed to have enough FDLs to provide burst contention resolution and there is no contentions in FDLs. [11] illustrates another possible node structure with FDLs, but it only shows the impact of the maximum FDL delay on the network performance. The aim of our paper is to compare two different node structures in OBS networks with FDLs; furthermore, we explore the roles of two parameters of the FDL buffer, namely, the granularity and maximum delay, in improving network performance under different reservation schemes. The remainder of this paper is organized as follows. In Section II, we briefly review optical burst contention resolutions using FDLs, and then we present the parameters of dimensioning FDLs and compare two different node structures with FDLs. Section III shows numerical results on the impact of dimensioning FDLs on the network performance. Finally, we conclude in Section IV. 


\section{BURST CONTENTION RESOULTIONS USING FDLS}

\section{A. General OBS networks with FDLs}

In OBS networks, edge nodes aggregate packets and assemble them into data bursts based on certain assembling algorithm; control packets containing the length of corresponding data bursts, routing information, etc., are also generated. A control packet is sent out in advance along a predetermined route, passing through multiple core nodes until it reaches the destination edge node. We denote the time gap between sending out the control packet and the data burst at source node as the initial offset. Since a control packet needs to configure switches and reserves wavelength for the data burst at each node, the minimum value of the initial offset $\left(T_{\text {off }}\right)_{0}$ is the sum of the control packet processing time $\left(T_{c p}\right)_{i}$, at each node along the route and the switch setup time ${ }_{(T s w)}[1]$, i.e.,

$$
\left(T_{\text {off }}\right)_{0}=\sum_{i=1}^{P}\left(T_{c p}\right)_{i}+T_{s w}
$$

At each intermediate node, a control packet is processed electronically in a first-come-first-served manner. After successfully reserving data channels for the incoming data burst, the offset is updated according to the number of remaining hops to the destination; then the control packet is regenerated and sent to the downstream node.

Although optical buffers are not mandatory in OBS networks, studies show that using FDLs as optical buffers can effectively improve the network performance [9]. In an OBS network with FDLs, when a control packet cannot successfully reserve a wavelength at an output port for its corresponding data burst, it will try to reserve the available FDL with the shortest delay instead. There are two different node structures in OBS networks with FDLs: one is that each link has a dedicated FDL module [8], and we call it FDL share per link; the other is that each node has only one FDL module to be shared by all links [11], and we call it FDL share per node. The difference between FDL share per link and FDL share per node mainly lies in the hardware cost and the efficiency of FDL utilization. FDL share per node needs much less single FDL elements, and much less wavelength converters. But the tradeoff is that it needs more ports in the optical switch. Since wavelength converters are expensive, in terms of minimizing hardware cost, FDL share per node is a better choice since it is less bulky and utilizes FDLs more efficiently than FDL share per link. The FDL module can be designed in various ways, but generally they can be categorized into two basic structures [8], as shown in Figure 1. One is that a desired delay is obtained by linearly increasing number of single FDL elements, as shown in Figure 1(a); the other consists of multiple FDL stages, which are connected by wavelength-sensitive switches. We denote $B$ as the maximum delay of FDL buffer and $b$ as the single fiber delay element, which is also named as granularity of FDL buffer. Both structures of the FDL module can provide discrete delay from $b$ to $B$ with increasing step of $b$. The difference is that the first type is simpler; but the second is more efficient and powerful [8]. In our paper, we focus on the optimal dimensioning of FDLs and we can assume a linear relationship

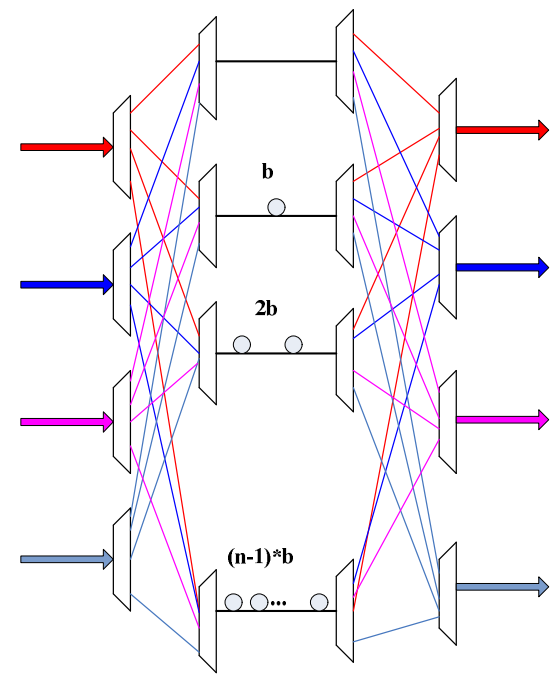

(a)

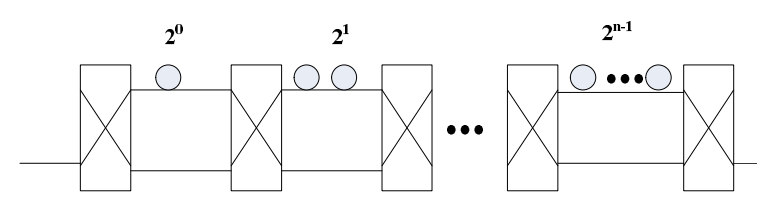

(b)

Figure 1. Two general stuctures of Fiber-delay-line (FDL) module

between the maximum delay and the granularity of the FDL buffer, i.e. we assume that the total number of $b$ to provide the maximum delay $B$ is $M$; with a fixed value of $\mathrm{b}$, increasing $M$ will linearly increase the maximum delay $B$.

\section{B. Reservation Schemes}

In general, in OBS networks with FDLs, control packets are first-come-first-served. When a control packet cannot successfully reserve a wavelength on an output port in a node of an OBS network with FDLs, it will try to reserve the shortest available FDL instead. Since a contended data burst can be delayed in an FDL with different wavelengths, each physical FDL with delay $D$ can be treated as $k$ virtual FDLs $D(k)$, in which $k$ is the number of wavelengths in each fiber link [8]. Figure 2 illustrates the chronological sequence of burst contention resolution using FDLs. We consider a scenario in which a node has one output port with a single wavelength and a single FDL with fixed delay $T_{b}$. Suppose a new control packet arrives at the current node $i$, and it attempts to reserve a wavelength in an output port for its data burst from $t_{s t}$ to $t_{e n}$ and the corresponding offset time is $\left(T_{o f f}\right)_{i}$. The reservation request for time interval $\left(t_{s t}, t_{e n}\right)$ fails since it is blocked by an already reserved data burst, shown in Figure 2(a). We name the time interval during the contention of two bursts the blocking time, i.e., $t_{2}-t_{s t}$ in Figure 2(a). However, a buffer is available during data burst transmission time $\left(t_{s t}, t_{e n}\right)$. Therefore, the data burst can be buffered and the new data burst transmission time becomes $\left(t_{s t}+T_{b}, t_{e n}+T_{b}\right)$, as shown in Figure 2(b). The detail of the scheduling algorithm to resolve burst contention by FDLs is given as follows. 
Set the FDL buffer with the maximum delay $B$. The granularity of an FDL buffer is $b$. We calculate the blocking time between the new incoming data burst and all scheduled data bursts on all data channels. According to the blocking time, we get the minimum delay to buffer the new data burst, min_blocking. Due to discrete FDL delay, the minimum FDL delay needed is $D$,

$$
D=\left\lceil\frac{\text { min_blocking }}{b}\right\rceil \times b
$$

in which $\lceil x\rceil$ is the smallest integer equal to or bigger than $x$.

Step 1: check if the required $D$ is bigger than the maximum delay $B$

If $D \leq B$, go to Step 2;

If $D>B$, drop the incoming data burst

Step 2: check if the physical FDL with delay $D$ is available

Use the scheduling algorithm LAUC-VF to check each virtual delay $D(i)$ is available for the incoming data burst.

If any virtual $D(i)$ is available, reserve the time interval $\left(t_{s t}+D, t_{e n}+D\right)$ in the output port and simultaneously reserve virtual FDL $D(i)$ for the incoming data burst with time interval $\left(t_{s t}, t_{e n}\right)$

If none of the virtual $D(i)$ is available, the incoming data burst is dropped.

After the control packet has successfully reserved the FDL and data channel for the contended data burst, the control packet is updated and sent to the downstream node. According to the instant of sending out the control packet, there are two options: one option is that control packet is electronically buffered with delay $D$ to keep the same offset as the scenario without FDLs [1,2,9-11]; the other option is to send out control packet immediately after its processing [12], as shown in Figure 3. Most previous work [1,2,9-11] note that a burst with longer offset time has lower dropping probability. FDLs provide contention resolutions and help to improve the performance in extended-offset QoS schemes. In order to keep the burst priority unchanged, the corresponding burst offset value must be the same as in the scenario without FDLs. For example, as shown in Figure 3(a), a control packet arrives at node $i$ at time $t_{1}$. This control packet contains the information that its data burst will arrive at time $t_{s t}$ with offset $\left(T_{\text {off }}\right)_{i}$. But it finds that contention occurs between time period $\left(t_{s t}, t_{e n}\right)$ in the data channels at the output port. If the data burst can be scheduled after delay $T_{b}$ and FDL with delay $T_{b}$ is available, the control packet will reserve time period $\left(t_{s t}^{\prime}, t_{e n}^{\prime}\right)$ for the data burst. Since the data burst is delayed by $T_{b}$, the control packet has to be delayed by $T_{b}$ simultaneously. This means that the control packet can not be sent to the downstream node after finishing its processing at time $t_{c p}$, and it has to be electronically buffered. For the second option, as shown in

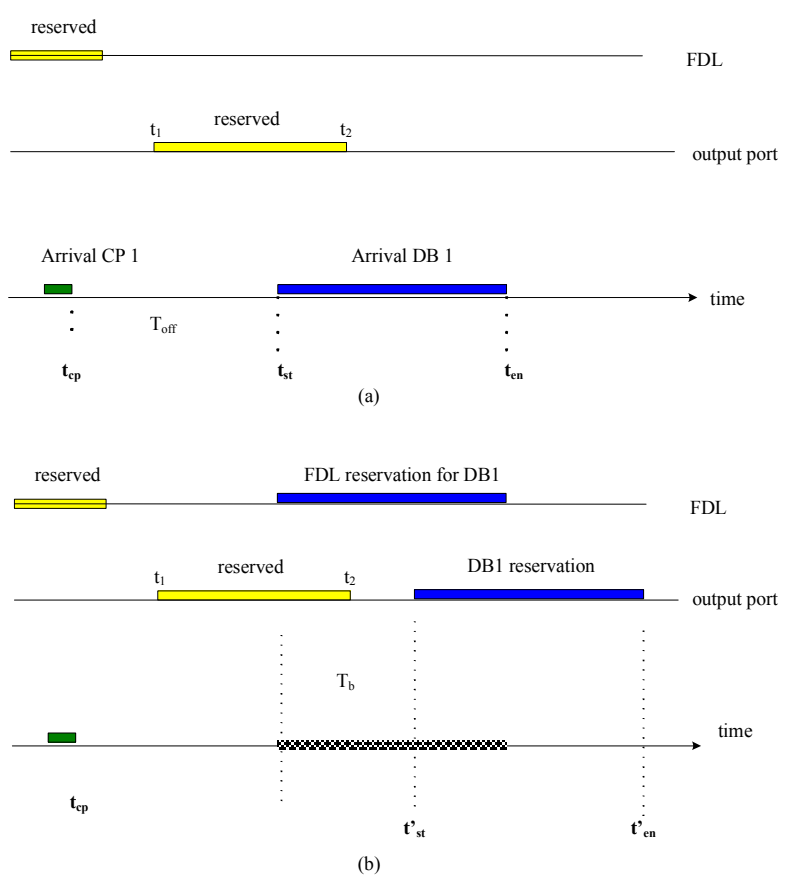

Figure 2. Chronological sequence of burst contention resolution using FDLs in a node with single wavelength and single FDL.

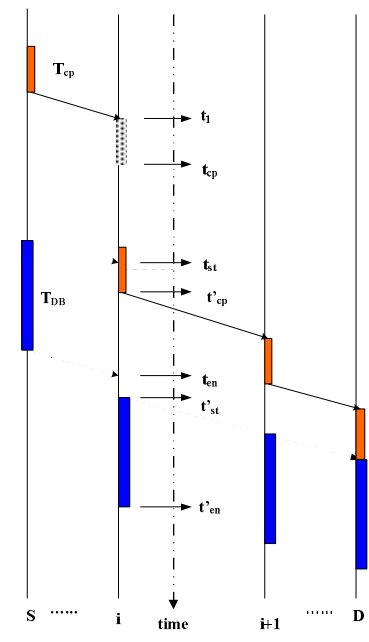

(a)

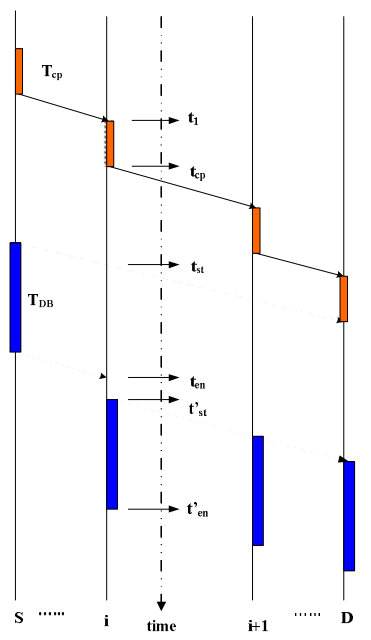

(b)
Figure 3. Two reservation schemes after the control packet reserves an FDL to resolve burst contention: (a) general OBS with FDLs; (b): dynamic offset scheme.

Figure 3(b), the control packet is instantly sent to the downstream node after it successfully reserves the data channel and FDL. Offsets at all downstream nodes will be dynamically changed according to the data burst delay at node $i$. The drawback of this reservation scheme is that it can not be implemented to provide QoS since changing the offset will change the priority of the data burst. We will investigate the problem of dimensioning FDLs with these two different reservation schemes in Section III. 


\section{PERFORMANCE EVALUATION}

We use simulations to investigate the problem of dimensioning FDLs in OBS networks. We study two types of network topologies: regular and irregular network topology. For the regular topology, an $8 \times 8$ Manhattan Street Network (MSN) is used. Each fiber link contains one channel for control packets and four wavelengths as data channels. For irregular topology, we use NSFNET, which is shown in Figure 4. We assume each link has one dedicated wavelength as the control channel and ten wavelengths for carrying data bursts in NSFNET. The input network traffic is in the form of data bursts. Following the assumption in [4], the data burst traffic and control packet traffic follow Poisson distribution, and data burst length is an exponential distribution. The average control packet processing time is assumed to be one tenth of the average data burst transmission time. Since we are only interested in the performance related to data bursts, we also assume there is no control packet loss on the control channel. Each node in the topology may be an edge node or a core node, and full wavelength conversion capability is available at each node. When a new data burst arrives at an edge node, it randomly chooses a destination from the rest of the nodes in the network and the shortest path routing algorithm is used. The scheduling algorithm is LAUC-VF. The normalized load offered to a node is defined as the ratio of the average data burst transmission time to the inter-arrival time normalized by the number of channels per link. All nodes are assumed to receive the same offered load in the network. Each node has same the FDL buffer and the FDL delay unit is normalized to the average data burst transmission time. In the following simulations, we use two different reservation schemes to study the problem of dimensioning FDLs: one is the reservation scheme used in general OBS networks in FDLs, in which a control packet is electronically buffered for the same delay as the data burst to keep the offset unchanged in the scenario of using FDLs as the contention resolution ( $S O$ scheme); another is dynamic offset $(D O)$, in which the control packet is not buffered and offset is updated dynamically at each node along the route.

Figures 5 and 6 show the role of maximum FDL delay on the network performance with different network topologies under different reservation schemes. The delay of a single FDL element, i.e., the granularity, is set to be 0.1 . We observe that, either in NSFNET or in MSN, network performance in terms of burst loss probability can be greatly improved with limited FDL delay. For example, in Figure 5, using FDLs with maximum delay of 2 units, the network performance in both reservation schemes improves $44.5 \%$ compared to the scenario without FDLs when the offered load is 0.6. From Figures 5 and 6 , we can also observe that, if the maximum delay of FDLs is greatly increased, the network performance only improves slightly. In other words, network performance can only be effectively improved with limited maximum FDL delay. The third observation is that using $D O$ can more

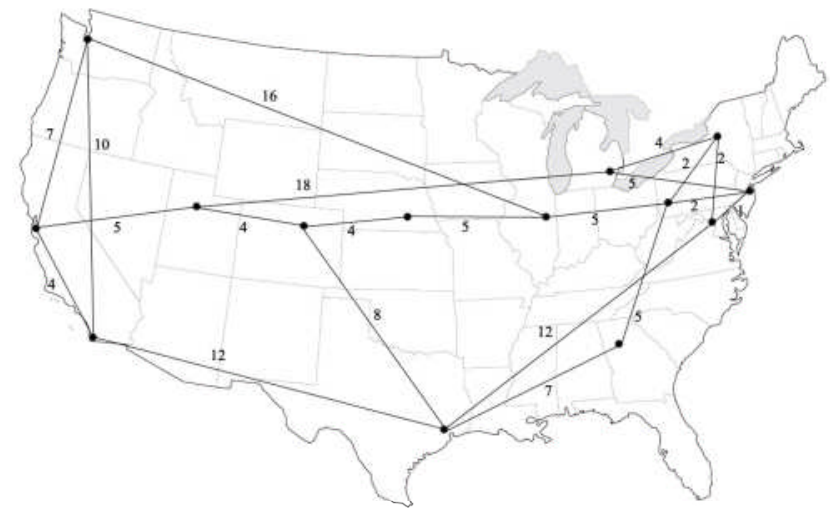

Figure 4. The NSFNET topology, 1991 (the original map is available at ftp.uu.net/inet/maps/nsfnet/).

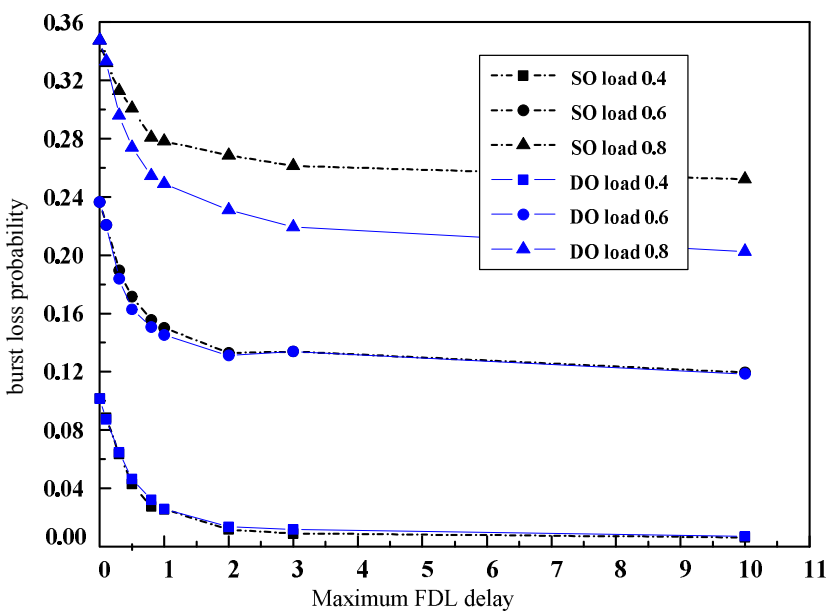

Figure 5. The impact of maximum FDL delay on network performance in NSFNET using different reservation schemes: reservation scheme in general OBS networks with FDLs is donated as SO, dynamic offset scheme is donated as $D O$.

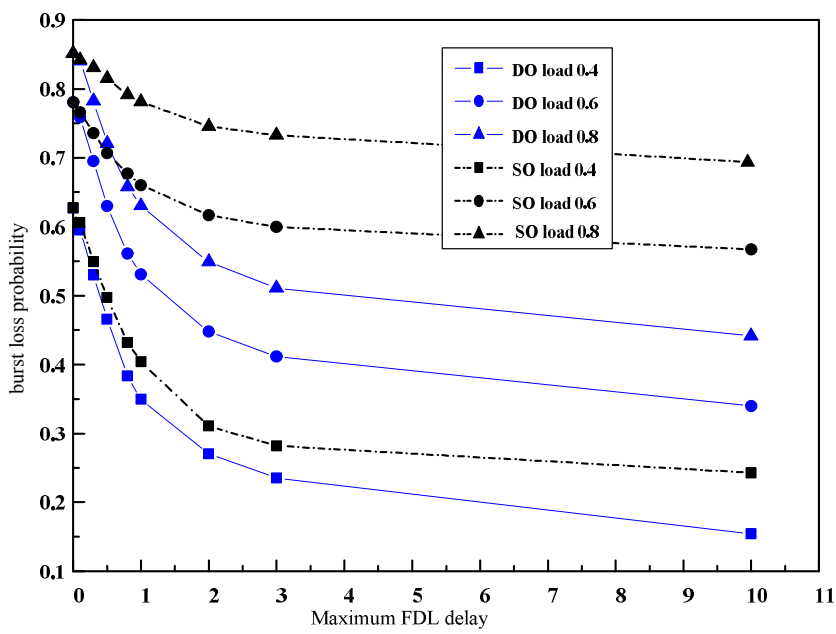

Figure 6. The impact of maximum FDL delay on network performance in MSN using different reservation schemes. 
effectively improve the network performance than the $S O$ scheme. For example, as in Figure 5 for NSNET with the maximum FDL delay being 2, the performance improvements using $D O$ and $S O$ are almost the same when the network load is 0.4 or 0.6 . However, when the network load is $0.8, D O$ can get $70 \%$ improvement and $S O$ can get $23 \%$ improvement with reference to the scenario without FDLs. In Figure 6 for MSN, the performance improvement of $D O$ is much larger than $S O$.

Figures 7 and 8 study the role of FDL granularity on network performance improvement. We set the maximum delay of FDLs to be 1 unit. Increasing the number of single fiber elements, the granularity of FDLs becomes smaller. We observe, in both NSNET and MSN, using DO or SO, partitioning FDLs into smaller elements cannot effectively improve network performance. In other words, with a certain maximum delay, the granularity of FDL buffer has little impact on the network performance in terms of burst loss probability.

\section{CONCLUSIONS}

We investigate two different node structures with FDLs; FDL share per link and share per node. Then, we analyze the roles of maximum FDL delay and FDL granularity on network performance improvement in terms of burst loss probability. By running simulations in an irregular network topology and a regular network topology, we find network performance can be effectively improved with limited FDL delay, but greatly increasing the maximum FDL delay and using a finer FDL granularity cannot effectively improve network performance. We also observe dynamic offset can get more performance improvement than general OBS networks with FDLs.

\section{ACKNOWLEDGMENT}

This research is supported in part by Independent Innovation Foundation of Shandong University (Project No. 2009TS105) and WEIHAI Science and Technology Development Plan (Project No. 2007063). The authors thank help from Prof. Victor Li, The University of Hong Kong, Dr. C.Y. Li and Prof. P.K.A. Wai, The Hong Kong Polytechnic University.

\section{REFERENCES}

[1] C. Qiao and M. Yoo, "Optical burst switching (OBS) - a new paradigm for an optical Internet," Journal of High Speed Networks, vol. 8, no. 1, pp. 69-84, 1999.

[2] J. $\mathrm{Xu}, \mathrm{C}$. Qiao, J. Li, and G. Xu, "Efficient channel scheduling algorithms in optical burst switched networks," in Proceedings of IEEE INFOCOM 2003, 3ed, pp. 2268-2278, 2003.

[3] J. Ramamirtham, J. Turner, and J. Friedman, "Design of wavelength converting switches for optical burst switching," IEEE Journal on Selected Arears in Communications, vol. 21, no. 7, pp. 1122-1132, 2003.

[4] Y. Chen, H. Wu, D. Xu, and C. Qiao, "Performance analysis of optical burst switched node with deflection routing," in Proceedings of IEEE ICC 2003, 2ed, pp. 1355-1359, 2003.

[5] A. Detti, V. Eramo, and M. Listanti, "Performance evaluation of a new technique for IP support in a WDM optical network: optical composite burst switching (OCBS)," Journal of Lightwave Technology, vol. 20, no. 2, pp. 154-165, 2002.

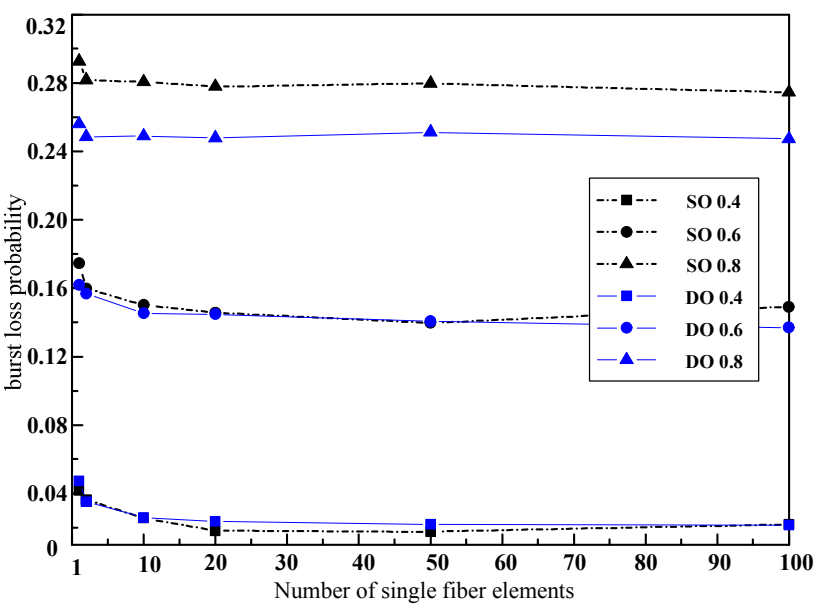

Fig. 7. The impact of FDL granularity on the network performance in NSFNET using different reservation schemes.

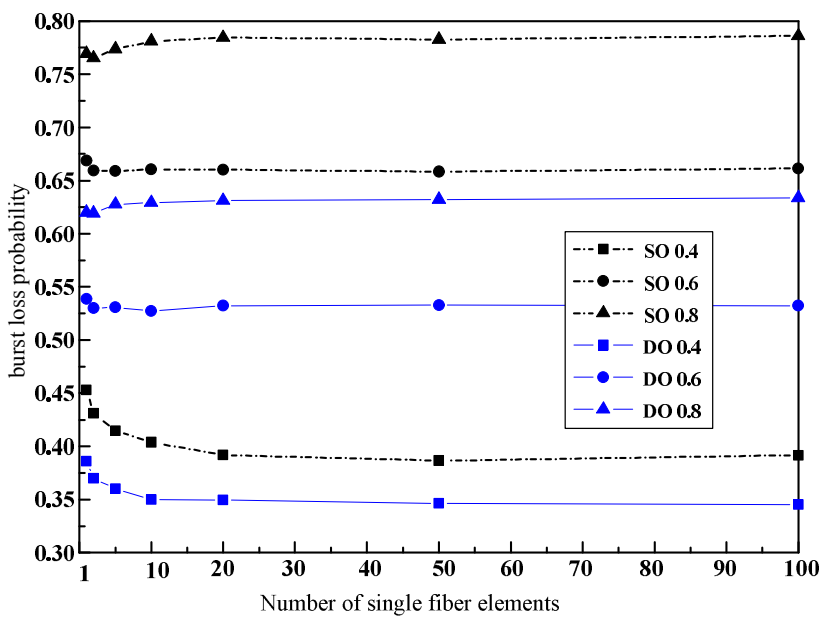

Fig. 8. The impact of FDL granularity on the network performance in MSN using different reservation schemes.

[6] V. A. Vokkarane, J. P. Jue, and S. Sitaraman, "Burst segmentation: an approach for reducing packet loss in optical burst switched networks," in Proceedings of IEEE ICC 2002, 5ed, pp. 2673-2677, 2002.

[7] V. M. Vokkarane and J. P. Jue, "Prioritized burst segmentation and composite burst-assembly techniques for QoS support in optical burstswitched networks," IEEE Journal on Selected Arears in Communications, vol. 21, no. 7, pp. 1198-1209, 2003.

[8] M. Yoo, C. Qiao, and S. Dixit, "QoS performance of optical burst switching in IP-over-WDM networks," IEEE Journal on Selected Arears in Communications, vol. 18, no. 10, pp. 2062-2071, Oct. 2000.

[9] X. Lu and B. L. Mark, "Performance modeling of optical-burst switching with fiber delay lines," IEEE Transactions on Communications, vol. 52, no. 12, pp. 2175-2183, 2004.

[10] S. Tan, G. Mohan, and K. Chua, "Burst rescheduling with wavelength and last-hop FDL reassignment in WDM optical burst switching networks," in Proceedings of IEEE ICC 2003, 2ed, pp. 1448-1452, 2003.

[11] Y. Xiong, M. Vandenhoute, and H. C. Cankaya, "Control architecture in optical burst-switched WDM networks," IEEE Journal on Selected Arears in Communications, vol. 18, no. 10, pp. 1838-1851, 2000.

[12] G. M. Li, C. Y. Li, Victor O.K. Li, P.K.A. Wai, "Novel reservation schemes in optical burst switching networks with fiber delay lines," in Proceedings of CHINACOM 2008, pp.454-458, 2008. 